\title{
Open Charm Tomography of Cold and Hot Nuclear Matter
}

\author{
Ivan Vitev \\ Los Alamos National Laboratory, Theoretical Division and Physics Division, Los Alamos, NM 87544, USA
}

\author{
Received on 23 December, 2006
}

\begin{abstract}
We identify the nuclear effects that modify the cross sections for open heavy flavor production in protonnucleus and nucleus-nucleus collisions. In p+A reactions, we calculate and resum the coherent nuclear-enhanced power corrections from the final-state parton scattering in the medium. We find that single and double inclusive open charm production can be suppressed as much as the yield of neutral pions from dynamical high-twist shadowing. Effects of energy loss in p+A collisions are also investigated. These lead to significantly weaker transverse momentum dependence of the nuclear attenuation and give a sizable contribution to the forward rapidity hadron suppression. In A+A reactions we revisit the question of the measured large heavy flavor quenching at RHIC. We derive the collisional broadening of the heavy meson's transverse momentum and the distortion of its intrinsic light cone wave function. The medium-induced dissociation probability of heavy mesons is shown to be sensitive to the opacity of the quark-gluon plasma and the time dependence of its formation and evolution. In contrast to previous results on heavy quark modification, our approach predicts suppression of $B$-mesons comparable to that of $D$-mesons at transverse momenta as low as $p_{T} \sim 10 \mathrm{GeV}$. It allows for an improved description of the large attenuation of non-photonic electrons in central Au+Au reactions at RHIC. Preliminary results in the implementation of collisional and radiative energy loss in a Langevin simulation approach to heavy quark diffusion and attenuation are also presented.
\end{abstract}

Keywords: Open heavy flavor

\section{NUCLEAR EFFECTS ON OPEN HEAVY FLAVOR PRODUCTION IN P+A COLLISIONS}

Relativistic heavy ion collisions are, at present, the best laboratory where theoretical techniques for describing the manybody QCD dynamics in the high energy limit can be developed. Because of its quantitative importance for heavy ion phenomenology, the theory of final-state radiative energy loss in the quark-gluon plasma has been a natural focus of perturbative QCD studies $[1,2]$. In contrast, initial-state energy loss in cold nuclei has not been discussed so far. We use the formal recurrence relation (GLV) approach to multiple parton scattering to find the complete solution to the problem of mediuminduced gluon emission from jets traversing cold nuclear matter [3]. The differential bremsstrahlung spectrum, where the Landau-Pomeranchuk-Migdal destructive interference effects are fully accounted for, is calculated for three different cases: 1) generalization of the incoherent Bertsch-Gunion solution for asymptotic on-shell jets; 2) initial-state energy loss of incoming jets that undergo hard scattering; 3 ) final-state energy loss of jets that emerge out of a hard scatter. Our analytic solutions are given as an infinite opacity series which represents cluster expansion of the sequential multiple scattering. These new solutions allow, for the first time, direct comparison between initial- and final-state energy loss in cold nuclei. To first order in opacity numerical results are shown in Fig. 1 for light, charm and bottom quarks and for medium parameters $L=5 \mathrm{fm}, \lambda_{g}=1 \mathrm{fm}$ and $\mu=0.35 \mathrm{GeV}$. The differences between initial- and final-state energy loss arise from the differences in the interference pattern of the Bertsch-Gunion and vacuum bremsstrahlung, determined by the hard scattering boundary conditions. Contrary to the naive assumption, we demonstrate that energy loss in cold nuclear matter can be large, $\Delta E / E \simeq 5 \%$ for light quarks in the $E \rightarrow \infty$ limit. As shown in Fig. 1, $\Delta E_{\text {initial }}>\Delta E_{\text {final }}$ and its path length depen- dence is linear, $\propto L[3]$, as opposed to final state energy loss, $\propto L^{2}[2]$.

We first investigate the effect of the multiple soft scatterings that precede and follow the hard partonic interaction in $\mathrm{p}+\mathrm{A}$ reactions. In deep inelastic scattering, in the framework of the QCD factorization approach, shadowing arises

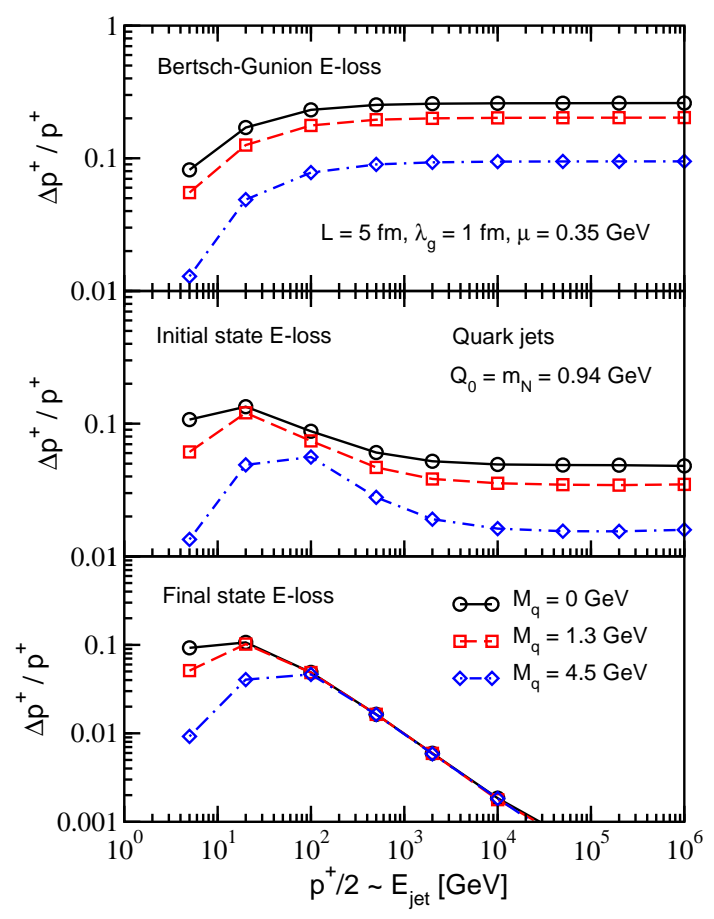

FIG. 1: Mass dependence of the fractional energy loss $\Delta E / E$ verses the jet energy $E_{\text {jet }}$. We studied massless quarks, $M_{q}=0 \mathrm{GeV}$, charm quarks $M_{c}=1.3 \mathrm{GeV}$ and bottom quarks $M_{b}=4.5 \mathrm{GeV}$. 


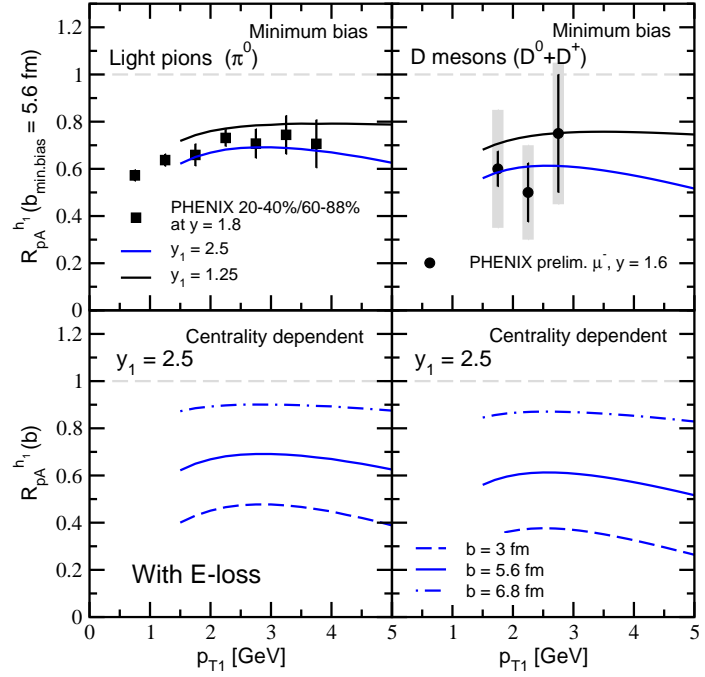

FIG. 2: Nuclear modification of single inclusive $\pi^{0}$ (left panels) and $D^{0}+D^{+}$mesons (right panels) in $\mathrm{d}+\mathrm{Au}$ collisions at RHIC. Cold nuclear matter energy loss is taken into account in the theoretical calculations. Data is from PHENIX [6, 7].

from the coherent final-state rescattering of the struck, small $x_{b}$ parton from the nucleus. Dynamical calculations of such nuclear-enhanced power corrections have been generalized to hadronic collisions for massless [4] and massive [5] final-state partons. High-twist shadowing leads to suppression of the single- and double-inclusive hadron production that increases with rapidity and centrality but disappears at high $Q^{2}$ [4]. While it can give a contribution to the low $p_{T}$ attenuation of the particle production cross sections, its effects are negligible at $p_{T} \geq 5 \mathrm{GeV}$. Theoretical calculations, constrained by DIS data, underpredict [5] the suppression of both light and heavy mesons [6, 7].

Having completed the derivation of the magnitude of cold nuclear matter energy loss [3] and used constraints from forward rapidity $\pi^{0}$ data from STAR and lower $\sqrt{s}$ data from NA35 we are able to present improved theoretical calculation in Fig. 2 [5]. The top panels show suppression of the single-inclusive hadron production rates in $\mathrm{d}+\mathrm{Au}$ reactions versus $p_{T}$ for rapidities $y_{1}=1.25$ (smaller attenuation) and $y_{1}=2.5$ (larger attenuation) at $\sqrt{s_{N N}}=200 \mathrm{GeV}$. The bottom panels show impact parameter dependence of the calculated nuclear modification for central, $b=3 \mathrm{fm}$, minimum bias, $b_{\text {min.bias }}=5.6 \mathrm{fm}$ and peripheral, $b=6.8 \mathrm{fm}$, collisions. Very good agreement between the QCD theory incorporating cold nuclear matter effects and the PHENIX measurement of muons coming from the decay of light hadrons and heavy flavor is achieved $[6,7]$.

\section{NUCLEAR EFFECTS ON OPEN HEAVY FLAVOR PRODUCTION IN A+A COLLISIONS}

The detailed suppression pattern of high transverse momentum hadrons is an important experimental signature of the quark-gluon plasma creation in heavy ion collisions [1]. Jet quenching for light mesons, such as $\pi, K$ and $\eta$, at RHIC is well explained by radiative energy loss calculations [8, 9]. In contrast, the measured suppression of single non-photonic electrons $[10,11]$ is too large when compared to QCD heavy quark energy loss predictions [12]. The cause of this disagreement is readily identified as the small quenching of $B$-mesons, which dominate the high- $p_{T}$ single electron yields.

It is usually assumed that the hard jet hadronizes in vacuum, having fully traversed the region of dense nuclear matter, $L_{T}^{\mathrm{QGP}} \leq 6 \mathrm{fm}$, and lost energy via radiative and collisional processes. In Ref. [13] we examined the validity of this assumption for different species of final-state partons and decay hadrons. For a $p_{T}=10 \mathrm{GeV}$ pion at mid-rapidity $\tau_{\text {form }} \approx 20 \mathrm{fm} \gg L_{T}^{\mathrm{QGP}}$, consistent with the jet quenching assumptions $[1,8]$. In contrast, $B$ - and $D$-mesons of the same $p_{T}$ have formation times $\tau_{\text {form }} \approx 1.6,0.4 \mathrm{fm}$, respectively, $\ll L_{T}^{\text {QGP }}$. Therefore, at the finite $p_{T}$ range accessible at RHIC and LHC a conceptually different approach to the description of $D$ - and $B$-meson quenching in $\mathrm{A}+\mathrm{A}$ collisions is required, when compared to light hadrons. Motivated by this finding, in the framework of the GLV theory, we derived the collisional dissociation probability of heavy mesons in the QGP [13]. The dynamics of open heavy flavor production and modification is represented by a set of coupled rate equations that describe the competition between $b$ - and $c$-quark fragmentation and $D$ - and $B$-meson dissociation. We solve these system of coupled rate equations numerically, using the same initial soft gluon rapidity density $d N^{g} / d y$ as in the calculation of the $\pi^{0}$ quenching [8] in central $\mathrm{Au}+\mathrm{Au}$ and $\mathrm{Cu}+\mathrm{Cu}$ collisions at RHIC. Contrary to calculations that emphasize radiative and collisional heavy quark energy loss [12], QGP-induced dissociation predicts $B$-meson suppression comparable to or larger than that of $D$-mesons at transverse momenta as low as $p_{T} \sim 10 \mathrm{GeV}$ [13]. The heavy meson spectra are decayed into electrons $\left(e^{+}+e^{-}\right)$using the PYTHIA event generator. Our results are shown in Fig. 3 and the predicted $R_{A A}\left(p_{T}\right)$, which does not neglect the large $B$-meson contribution, de-

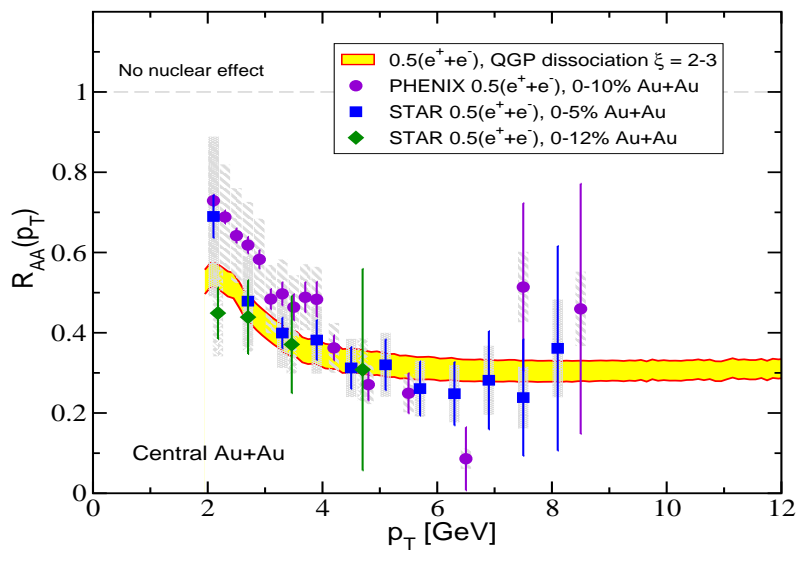

FIG. 3: Suppression of inclusive non-photonic electrons from $D$ and $B$-meson spectra softened by collisional dissociation in central $\mathrm{Au}+\mathrm{Au}$ collisions. Data on non-photonic electron quenching from PHENIX [10, 16] and STAR [11, 14, 15] is also shown. 


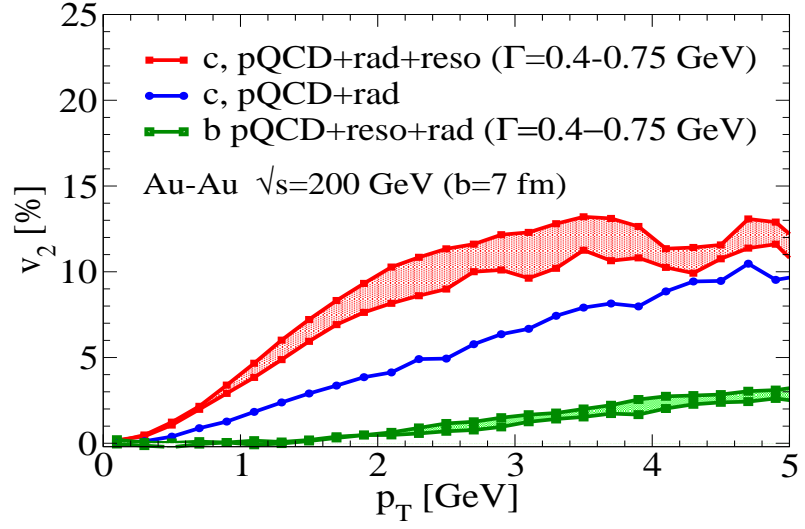

FIG. 4: Elliptic flow $v_{2}$ for heavy c- and b-quarks from collisional and radiative energy loss and quark-resonance interactions [18]. Note that $v_{2}(b) \ll v_{2}(c)$. For charm quarks, the PQCD $\Delta E$ contribution is shown separately.

scribes well the most recent heavy flavor quenching measurements at RHIC [14-16].

Large nuclear suppression and elliptic flow $v_{2}$ can also be obtained in Langevin simulations of heavy quark diffusion. It has been argued that strong coupling between the $c$ - and $b$-quarks and the QGP medium may be generated via quark-resonance interactions near the phase QCD transition, $T \sim T_{c}$ [17]. It is, therefore, important to study the interplay between such non-perturbative effects and the radiative and collisional heavy quark energy loss. We evaluate the contribution of these processes to the drag and diffusion coefficients, $A_{i}=\left(1 / p_{i}\right)\left\langle\delta p_{i}\right\rangle / \delta t$ and $B_{i j}=(1 / 2)\left\langle\delta p_{i} \delta p_{j}\right\rangle / \delta t$, which are then applied in a relativistic Fokker-Planck equation. Preliminary results on the $p_{T}$-dependent azimuthal asymmetry for minimum-bias $\mathrm{Au}+\mathrm{Au}$ collisions are shown in Fig. 4. We note that collisional and radiative energy loss can give a large $\sim 10 \%$ elliptic flow at $p_{T}=5 \mathrm{GeV}$. Quark-resonance interactions are seen to also significantly contribute to heavy quark observables. We note that the generated $v_{2}$ for $b$-quarks is much smaller than that for $c$-quarks. Future Langevin simulations of $c$ - and $b$-quark diffusion should include momentum fluctuations beyond the Einstein's fluctuation-dissipation relation and the decay of the heavy quark spectra into $\left(e^{+}+e^{-}\right)$ for direct comparison to the non-photonic electron observables at RHIC [18].

\section{CONCLUSIONS}

In these proceedings, we discussed the multiple scattering effects that drive the cold nuclear matter attenuation of $D$-meson and light hadron production at forward rapidities, namely, high twist shadowing [4] and parton energy loss $[3,5]$. We emphasize that the GLV theory of multiple parton interactions in QCD matter has been used to obtain the full solution for the initial-state energy loss of jets in cold nuclei, a significant improvement relative to previous phenomenological treatments. Our results demonstrate that in the framework of the QCD collinear factorization dynamically generated and process-dependent nuclear effects can be incorporated to give a more reliable description of high- $p_{T}$ particle production in $\mathrm{p}+\mathrm{A}$ collisions $[6,7]$.

In $\mathrm{A}+\mathrm{A}$ reactions, motivated by the short formation times of heavy mesons, we developed a model of their QGP-induced collisional dissociation [13]. Good description [14-16] of the large quenching of the non-photonic electrons measured by PHENIX [10] and STAR [11] is achieved. A natural consequence of this new approach is that $B$-mesons are attenuated as much as $D$-mesons at transverse momenta as low as $p_{T} \sim 10 \mathrm{GeV}$. While we anticipate that a comparable description of the non-photonic electron quenching may be achieved when partonic energy loss is combined with quark-resonance interactions near the QCD phase transition in Langevin transport simulations [17], the hierarchy $R_{A A}(H(c)) \ll R_{A A}(H(b))$ will not be changed in the accessible transverse momentum range [18].

\section{Acknowledgements}

This work is supported by the United States Department of Energy under Contract No. DE-AC52-06NA25396 and the J. Robert Oppenheimer Fellowship of the Los Alamos National Laboratory.
[1] M. Gyulassy, I. Vitev, X. N. Wang, and B. W. Zhang, nuclth/0302077.

[2] M. Gyulassy, P. Levai, and I. Vitev, Nucl. Phys. B 594, 371 (2001).

[3] I. Vitev, Phys. Rev. C in press (2007), hep-ph/0703002.

[4] J. W. Qiu and I. Vitev, Phys. Lett. B 632, 507 (2006).

[5] I. Vitev, T. Goldman, M. B. Johnson, and J. W. Qiu, Phys. Rev. D 74, 054010 (2006).

[6] X.R. Wang [PHENIX Collaboration], J. Phys. G 32, S511 (2006).

[7] X. R. Wang [PHENIX Collaboration], AIP Conf. Proc. 842, 68 (2006); hep-ex/0603051.

[8] I. Vitev, Phys. Lett. B 639, 38 (2006).

[9] J. Lajoie, nucl-ex/0611002.
[10] A. Adare [PHENIX Collaboration], nucl-ex/0611018.

[11] B. I. Abelev et al. [STAR Collaboration], nucl-ex/0607012.

[12] S. Wicks, W. Horowitz, M. Djordjevic, and M. Gyulassy, nuclth/0512076.

[13] A. Adil and I. Vitev, Phys. Lett. B 649, 139 (2007).

[14] A. Suaide, J. Phys. G in press (2007), nucl-ex/0702035.

[15] C. Zhong [STAR Collaboration], J. Phys. G in press (2007), nucl-ex/0702014.

[16] R. Averbeck, "Quark Matter 2006" proceedings, J. Phys. G to be published

[17] H. van Hees and R. Rapp, Phys. Rev. C 71, 034907 (2005).

[18] I. Vitev, A. Adil and H. van Hees, J. Phys. G in press (2007), hep-ph/0701188. 